\title{
Clinical Profile of Anemia in Children
}

\author{
Venkatesh $\mathrm{G}^{1}$, Soubhagya Talawar ${ }^{2}$, Bela H Shah ${ }^{3}$ \\ , ${ }^{1}$ Ex Junior Resident, Department of Pediatrics, BJMC Ahmedabad, Gujarat, India \\ ${ }^{2}$ Ex Junior Resident, Department of Obstetrics and Gynecology, KIMS Hubli, Karnataka, India \\ ${ }^{3}$ Additional professor, Department of Pediatrics, BJMC Ahmedabad, Gujarat, India
}

\begin{abstract}
Anemia is considered as a worldwide problem, affecting all age groups. Anemia in children is one of the social health problems because the children have reduced exercise capacity, slower growth and impaired cognitive development. The present study was conducted to quantify the magnitude of anemia and its association with malnutrition.
\end{abstract}

Keywords: Anemia, magnitude of anemia, malnutrition.

\section{Introduction}

About half of the population in the developing countries has iron deficiency anemia. Preschool, school and adolescent children and women in childbearing age are at increased risk. Iron deficiency anemia affects $30 \%$ of the world population $[1,2]$. The prevalence of anemia among children under 5years of age is estimated to be about $20 \%$ in industrialized countries and 39\% in non-industrialized countries [3]. Iron deficiency anemia is a leading cause of morbidity and mortality worldwide [1]. In India, the national program for prevention and control of anemia focuses on pregnant women and young children less than 5years. However, the status of anemia in children is not well documented [1].

\section{Materials And Methods}

This study was conducted during the period November 2007 - May 2009 in BJMC Ahmedabad, Gujarat, India. Patients having severe nutritional anemia with hemoglobin $<7 \mathrm{gm} \%$ were evaluated.

Exclusion criteria: Patients having mild to moderate anemia, severe anemia due to hemolysis, malaria, aplastic anemia and patients collapsed due to congestive cardiac failure within 12 hours of admission were excluded.

Detailed history regarding symptoms and signs, diet and socioeconomic status of family and detailed anthropometry were recorded. Investigations for anemia and its causes i.e., peripheral smear, serum vitamin B12, serum folic acid, stool for worm infestation were done in all patients. The typing of anemia was done based on these reports. Hemoglobin was estimated by Sahli's method and expressed in gm\%, peripheral smear was stained by Leishman's stain, and PCV, MCV, MCH, MCHC and RDW were determined by automated cell counter. Normal values were taken as follows: PCV 35-45\%, MCV 77-95fl, MCH 25-33pg, MCHC 31-37gm/dl and RDW 14.5-18.5.Reticulocyte count was done by Brilliant crystal stain method, serum iron determination was done by Ramany's dipyridyl method, Total iron binding capacity was determined by Ramsay's method, serum vitamin B12 and folic acid was determined by Architect method. Results were analyzed by GraphpadInstat3 software program. $P$ value less than 0.05 was taken as statistically significant.

\section{Results}

A total of 9124 patients were admitted to pediatric ward at our institute from November 2007 - May 2009 , of which patients having anemia were $7412(81.2 \%)$, severe anemia were 202 ; prevalence of severe anemia being $2.2 \%$.

\subsection{Age wise distribution of patients with severe anemia - Fig. 1}

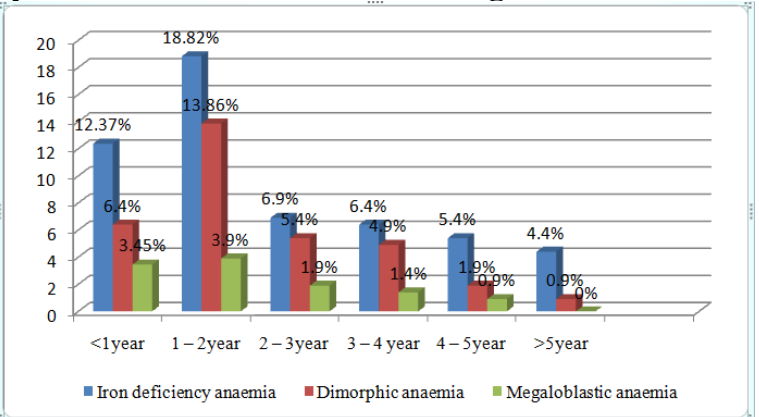

Figure 1: Age-wise distribution of patients with severe anemia 
It is observed from above table that all three types of anemia were more in 1-2years of age group.

\subsection{Sex wise distribution of anemia - Table -1}

Table -1: Sex-wise distribution of anemia

\begin{tabular}{|l|l|l|l|l|}
\hline & $\begin{array}{l}\text { Iron deficiency } \\
\text { anemia }\end{array}$ & $\begin{array}{l}\text { Megaloblastic } \\
\text { anemia }\end{array}$ & Dimorphic anemia & Total \\
\hline Male & $52(25.7 \%)$ & $37(18.3 \%)$ & $10(4.9 \%)$ & $99(49 \%)$ \\
\hline female & $58(28.7 \%)$ & $31(15.3 \%)$ & $14(6.9 \%)$ & $103(50.99 \%)$ \\
\hline
\end{tabular}

For iron deficiency anemia, incidence is more in females i.e., $28.7 \%$ against males $25.7 \%$ while for megaloblastic anemia incidence is more in males i.e., $18.3 \%$ against $15.3 \%$ in females and for dimorphic anemia incidence in females is more i.e. $6.9 \%$ against males $4.9 \%$ which is not statistically significant ( $\mathrm{P}$ value $>0.05)$

3.3. Prevalence of different types of anemia. Fig. 2

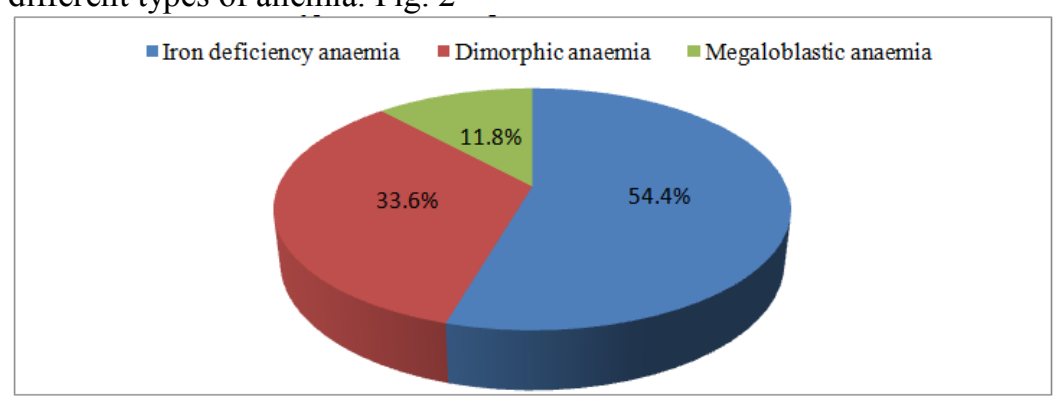

Figure -2: Prevalence of different types of anemia

In the current study iron deficiency anemia is most common followed by dimorphic anemia and megaloblastic anemia.

\subsection{Relationship between exclusive breastfeeding and anemia. Fig. 3}

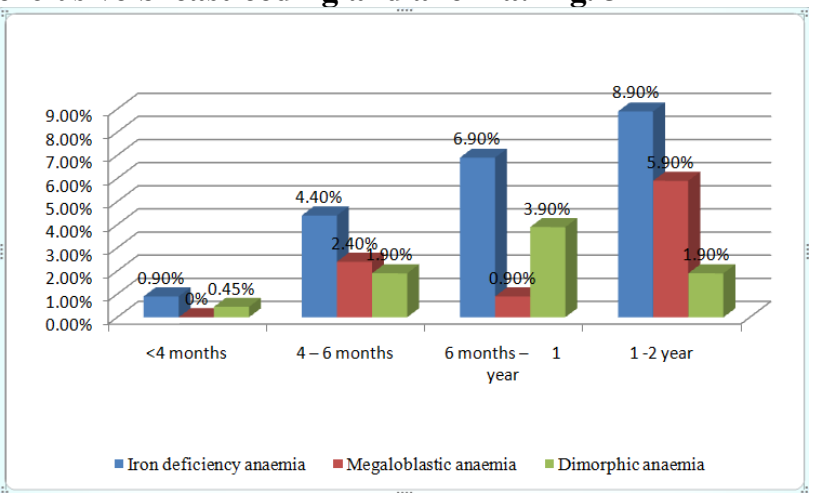

Figure - 3: Relationship between exclusive breastfeeding and anemia

In the present study, maximum numbers of patients are in $1-2$ year age group. Exclusive breast feeding up to 4-6 months helps in controlling anemia of infancy through breast milk. Although breast milk has very low iron content it has high bioavailability which is seen in present study.

\subsection{Symptoms}

Fever is seen in $59.4 \%$ of patients followed by weakness in $53.9 \%$ of patients, cough in $35.6 \%$, diarrhea in $16.3 \%$, breathlessness in $11.88 \%$, pica in $9.9 \%$, worm infestation in $8.9 \%$, vomiting in $7.9 \%$, tremor in $6.4 \%$ and blood in stool in $1.4 \%$.

\subsection{General examinations}

Pallor is seen in $100 \%$ of patients, vitamin deficiency in $54.4 \%$, knuckle pigmentation in $29.7 \%$, edema in $21.7 \%$ and koilonychia in $10.8 \%$. 


\subsection{Relation of $\mathrm{RBC}$ indices in anemia. Table -2}

Table -2: Relation of RBC indices in anemia

\begin{tabular}{|l|l|l|l|}
\hline Parameter & Iron deficiency anemia & Megaloblastic anemia & Dimorphic anemia \\
\hline $\mathrm{PCV} \downarrow$ & $110(54.4 \%)$ & $24(11.8 \%)$ & $68(33.6 \%)$ \\
\hline $\mathrm{MCV} \downarrow$ & $110(54.4 \%)$ & - & $30(14.8 \%)$ \\
\hline $\mathrm{MCV} \uparrow$ & - & $24(11.8 \%)$ & $38(18.8 \%)$ \\
\hline $\mathrm{MCH}$ Normal & - & - & $40(19.8 \%)$ \\
\hline $\mathrm{MCH} \downarrow$ & $110(54.4 \%)$ & $24(11.8 \%)$ & $28(13.8 \%)$ \\
\hline $\mathrm{MCHC}$ Normal & $16(7.9 \%)$ & $16(7.9 \%)$ & $32(15.8 \%)$ \\
\hline $\mathrm{MCHC} \downarrow$ & $94(46.5 \%)$ & $8(3.9 \%)$ & $36(17.8 \%)$ \\
\hline $\mathrm{RDW} \uparrow$ & $110(54.4 \%)$ & $8(3.9 \%)$ & $60(29.7 \%)$ \\
\hline $\mathrm{RDW} \downarrow$ & - & $16(7.9 \%)$ & $8(3.9 \%)$ \\
\hline
\end{tabular}

$\mathrm{PCV}$, packed cell volume; MCV, mean corpuscular volume; $\mathrm{MCH}$, mean corpuscular hemoglobin; $\mathrm{MCHC}$, mean corpuscular hemoglobin concentration; RDW, red cell distribution width.

In Iron deficiency anemia $\mathrm{PCV}, \mathrm{MCV}, \mathrm{MCHC}, \mathrm{MCH}$ are decreased and $\mathrm{RDW}$ is increased. In megaloblastic anemia PCV and $\mathrm{MCH}$ are decreased, MCV and MCHC are increased. In dimorphic anemia PCV is decreased in all patients and variation is seen in all other indices.

\subsection{Peripheral smear examination}

Microcytic hypochromic anemia is seen in $54.4 \%$, macrocytic hypochromic anemia is seen in $11.8 \%$ and dimorphic anemia is seen in $36.6 \%$ of patients.

\subsection{Relationship between anemia and PEM. Fig. - 4}

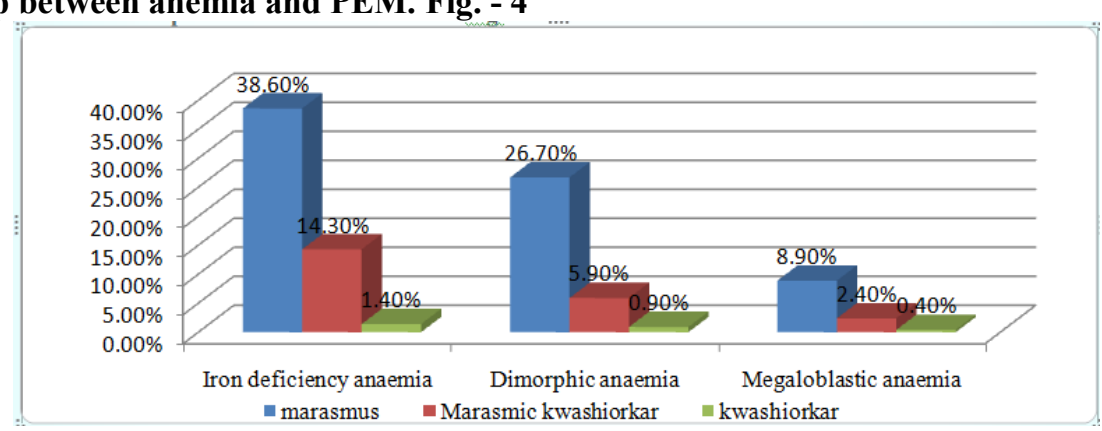

Figure - 4: Relationship between anemia and PEM

In iron deficiency anemia, patients in marasmus group are $38.6 \%$ against $14.3 \%$ in marasmic kwashiorkor. In dimorphic anemia, patients in marasmus group are $26.7 \%$ against $5.9 \%$ in marasmic kwashiorkor group. In megloblastic anemia $8.9 \%$ patients are in marasmus group as compared to $2.4 \%$ in marasmic kwashiorkor.

\subsection{Relation of socio economic class in anemia patients}

Most of the patients are in class V socioeconomic group i.e. $53.9 \%$, class IV $-24.2 \%$, class III $-14.8 \%$, class II $-1.9 \%$ and class I - $0 \%$. This is according to Kuppuswamy socioeconomic scale and this is in contrast to study conducted by Fadila A Assiri et al $(16,17)$.

\subsection{Stool for ova and cyst examination. Table -3}

Table -3 : Stool for ova and cyst examination

\begin{tabular}{|l|l|l|l|}
\hline Stool examination & Iron deficiency anemia & $\begin{array}{l}\text { Dimorphic } \\
\text { anemia }\end{array}$ & Megaloblastic anemia \\
\hline Positive & $32(15.8 \%)$ & $9(4.4 \%)$ & $8(3.9 \%)$ \\
\hline Negative & $78(38.6 \%)$ & $59(29.2 \%)$ & $16(7.9 \%)$ \\
\hline
\end{tabular}

Stool examination for ova and cyst was positive in $15.8 \%$ of iron deficiency anemia, $4.4 \%$ of dimorphic anemia and $3.9 \%$ of megaloblastic anemia. Stool examination was negative in $38.6 \%$ of iron deficiency anemia, $29.2 \%$ of dimorphic anemia and $7.9 \%$ of megaloblastic anemia. In present study $79 \%$ of patients presented with infection and $11.8 \%$ of patients presented with congestive cardiac failure due to severe anemia. 


\subsection{Treatment given for anemia. Table -4}

Table - 4: Treatment given for anemia

\begin{tabular}{|l|l|l|l|}
\hline Treatment & Iron deficiency anemia & Dimorphic anemia & Megaloblastic anemia \\
\hline Transfusion & $72(35.6 \%)$ & $43(21.2 \%)$ & $5(2.4 \%)$ \\
\hline Oral iron & $110(54.4 \%)$ & $68(33.6 \%)$ & $24(11.8 \%)$ \\
\hline Folic acid & - & $68(33.6 \%)$ & $24(11.8 \%)$ \\
\hline Methicobalamine & - & $68(33.6 \%)$ & $24(11.8 \%)$ \\
\hline
\end{tabular}

In present study $35.65 \%$ patients with iron deficiency anemia, $21.2 \%$ patients with dimorphic anemia and $2.4 \%$ patients with megaloblastic anemia were transfused packed cell volume. Remaining patients were managed by oral hematinics.

\subsection{Prevalence}

\section{Discussion}

Prevalence of anemia in our study was $81.2 \%$, which is in accordance to study conducted by Margaret F et al, whereas study conducted by Ahmed Mubarak et al [11] and Shally Awasthi et al [1], found prevalence of $37-38 \%$, study by Bijan Keikhaei et al [3] found prevalence of $43.9 \%$ and the study conducted by Peter R Dallman et al [4] showed prevalence of $6 \%$. Prevalence of severe anemia in current study was $2.2 \%$, which is in accordance to the study conducted by Shally Awasthi et al [1], whereas a study conducted by Job C.J.Calis et al [5] found prevalence of $12-29 \%$.

\subsection{Age wise distribution of anemia}

It is observed from our study that all three types of anemia were more in 1-2 years age group, which is in accordance to previous studies $[1,2,3,4,5,7,9,10,13,14]$. It may be due to poverty, maternal anemia, continued exclusive breast feeding beyond 6 months and improper complimentary diet. As the age advances there is decrease in the incidence of anemia probably due to introduction of proper feeding.

\subsection{Sex wise distribution}

There is no statistical significant difference between the two sexes in the current study, which is in accordance to previous studies [1,3,5,11,16], whereas study conducted by Neeraj Jain et al [8] found higher incidence of anemia in girls and Rebecca J Stoltzfus et al [12] found higher incidence of anemia in boys.

\subsection{Prevalence of different type of anemia}

Iron deficiency anemia is most common in our study followed by dimorphic anemia and megaloblastic anemia, which is in accordance to previous studies $[1,2,3,4,8,10,12,13,14,15]$.

There are several causes of iron deficiency anemia. The principal cause in children in developing countries is inadequate intake of usable iron, which is normally found in a well-balanced diet in the form of heme and nonheme iron. Several well controlled trials have been effective in reducing the prevalence of anemia in school-age children. Fortification is an attractive option for controlling iron deficiency anemia in countries where a significant number of groups are vulnerable to an ever increasing consumption of centrally processed foods [10].

\subsection{Relation between exclusive breast feeding and anemia}

Exclusive breast feeding up to 4-6 months helps in controlling anemia of infancy through breast milk which is seen in present study. This is in accordance to study conducted by Bijan Keikhaei et al [3].

\subsection{Symptoms and signs}

In the current study fever is the most common symptom followed by weakness, cough etc. This is in accordance to previous studies $[1,2,3,5,9,10,11]$. Pallor, vitamin deficiency was found in our study, which is in accordance previous studies $[2,5]$.

\subsection{Relation of RBC indices in anemia}

In Iron deficiency anemia PCV, MCV, MCHC, $\mathrm{MCH}$ are decreased and RDW is increased. In megaloblastic anemia PCV and MCH are decreased, MCV and MCHC are increased. In dimorphic anemia PCV is decreased in all patients and variation is seen in all other indices. This is in accordance to previous studies [3, 4]. In the current study microcytic hypochromic anemia is most common followed by dimorphic anemia and macrocytic hypochromic anemia; this is in accordance to the study conducted by Neeraj Jain et al [8].

\subsection{Relationship between anemia and PEM}

For iron deficiency anemia, patients in marasmus group are $38.6 \%$ against $14.3 \%$ in marasmic kwashiorkor. For dimorphic anemia, $26.7 \%$ patients are in marasmus group against $5.9 \%$ in marasmic 
kwashiorkor group. For megloblastic anemia, $8.9 \%$ patients are in marasmus as compared to $2.4 \%$ in marasmic kwashiorkor group. Studies to compare the results were not found in other articles. It is therefore concluded that association of protein energy malnutrition is widely prevalent in preschool children in India. There is an association between anemia and malnutrition with lower hemoglobin levels in the underweight and stunted children.

\subsection{Relation of socio economic class in anemia patients}

In the current study patients are in class $\mathrm{V}$ socioeconomic status, which is in accordance to previous studies [1, 2, 3, 8, 13]; but the study conducted by Ahmed Mubarak et al [11] found more cases in average socioeconomic status group.

\subsection{Stool for ova and cyst examination}

In the current study, stool examination for ova and cyst was positive in $15.8 \%$ of iron deficiency anemia, $4.4 \%$ of dimorphic anemia and $3.9 \%$ of megaloblastic anemia patients, which is in accordance to the previous studies [5, 8]. The study conducted by Imelda T Angeles et al [6] and Rebecca J Stoltzfus et al [12] found higher parasitic infection in children.

\subsection{Treatment}

In the current study $35.65 \%$ patients with iron deficiency anemia, $21.2 \%$ patients with dimorphic anemia and $2.4 \%$ patients with megaloblastic anemia were transfused packed cell volume. Remaining patients were managed by oral hematinics.

\section{Conclusion}

Anemia in association with malnutrition is widely prevalent in our country. So there is a need for urgent community participation strategies in the form of counseling the parents for child feeding practices, immunization and sickness recognition from the first year of life. Preventive measures for anemia control in children must be accompanied by measures to prevent underweight and stunting by focusing on integrated child feeding, health and environmental core measures.

\section{References}

[1] Shally Awasthi, Rohini Das, Tuhina Verma, Sheila Vir. Anemia and Undernutrition Among Preschool Children in Uttar Pradesh, India. Indian Pediatrics 2003; 40:985-990.

[2] Sarah E Cusick, Zuguo Mei, David S Freedman, Anne C Looker, Cynthia L Ogden, Elaine Gunter,et al. Unexplained decline in the prevalence of anemia among US children and women between 1988-1994 and 1999-2002 $2^{1-3}$. The American Journal of Clinical Nutrition 2008; 88: 1611-7

[3] Bijan Keikhaei, Khodamorad Zandian, Ali Ghasemi, Ramin Tabibi. Iron-deficiency anemia among children in southwest Iran. Food and Nutrition Bulletin $($ C 2007; vol.28, no.4:406-411, The United Nations University.

[4] Peter R Dallman, Ray Yip, Clifford Johnson. Prevalence and causes of anemia in the United States, 1976 to $1980^{1,2}$. The American Journal of Clinical Nutrition 1984; 39: 437-445.

[5] Job C.J.Calis, Kamija S. Phiri, E.Brian Faragher, Bernard J.Brabin, Imelda Bates, Luis E. Cuevas et al. Severe Anemia in Malawian Children .The New England Journal of Medicine 2008;358:888-99.

[6] Imelda T Angeles, Werner J Schultink, Paul Matulessi, Rainer Gross, Soemilah Sastroamidjojo. The American Journal of Clinical Nutrition 1993; 58:339-42.

[7] Ernesto Pollitt. Iron-Deficiency Anemia: Reexamining the Nature and Magnitude of the Public Health Problem. Journal of Nutrition 2001; 131:669S-675S.

[8] Neeraj Jain, Vibha Mangal Jain. Prevalence of anemia in school children. Medical Practice and Review Feb 2012; Vol.3 (1):pp.1-4.

[9] Margaret F. Gutelius. The Problem of Iron Deficiency Anemia in Preschool Negro Children. A.J. P. H. Feb 1969; Vol. 59, no.2:290-295.

[10] Mark Anthony Beinner, Joel Alves Lamounier. Food and Nutrition Bulletin (C) 2003; vol.24, no.3:268-274, The United Nations University.

[11] Ahmed Mubarak, Wael Fadel, Shibl Said, Mohamed Abu Hammer. Profile of Behavior and IQ in Anemia Children .International Journal of Neuropsychiatric Medicine, CNS Spectr. 2010; 15(12): ePub Ahead of Print.

[12] Rebecca J Stoltzfus, Hababu M Chwaya, James M Tielsch, Kerry J Schulze, Marco Albonico, Lorenzo Savioli. Epidemiology of iron deficiency anemia in Zanzibari schoolchildren: the importance of hookworms ${ }^{1-3}$. The American Journal of Clinical Nutrition 1997; 65: 153-9.

[13] Salvador Villalpando, Teresa Shamah, Juan A. Rivera, Yaveth Lara, Eric Monterrubio. Fortifying Milk with Ferrous Gluconate and Zinc Oxide in a Public Nutrition Program Reduced the Prevalence of Anemia in Toddlers ${ }^{1}$. The Journal of Nutrition 2006; 136: 2633-2637.

[14] Purnima Menon, Marie T. Ruel, Cornelia U. Loechl, Mary Arimond, Jean-Pierre Habicht, Gretel Pelto et al. Micronutrient Sprinkles Reduce Anemia among 9-to 24-Mo-Old Children When Delivered through an Integrated Health and Nutrition Program in Rural Haiti. The Journal of Nutrition 2007; 137: 1023-1030.

[15] Betsy Lozoff, Elias Jimenez, Abraham W. Wolf. Long-Term Developmental Outcome of Infants with Iron Deficiency. The New England Journal of Medicine 1991; 325: 687-94.

[16] Fadila A Assiri, Mona Al-Sumaie, Fatema Safar, Faisal Al-Saigh, Duaa Kalifa Hussain. Prevalence and associated factor of iron deficiency anemia among Kuwaiti children. Bull. Alex. Fac. Med. 42 No.2, 2006.

[17] Neeta Kumar, Neeru Gupta, Jugal Kishore. Kuppuswamy's socioeconomic scale: Updating Income Ranges for the year 2012. Indian Journal of Public Health, Vol. 56, issue 1, January-March, 2012. 